\title{
Using XML-coded Markovmodels to Build a Framework for Cost-Benefit Analysis in Cardiology
}

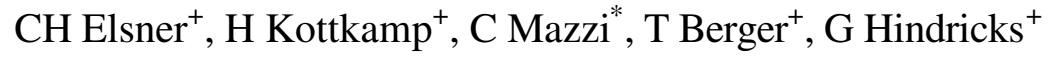 \\ ${ }^{+}$Heart Center Leipzig, Leipzig, Germany and " INSEAD Business School Fontainebleau, France
}

\begin{abstract}
Cost-Effectiveness Analysis becomes an important tool for Providers and Payers in establishing new therapeutic options for patients. Despite a huge amount of published Information on Evidence based Data on CostEffectiveness is available over Medical Databases (e.g. MedLine) there is a huge time gap in "time to provider" and "time to payer" of this information.

Objective of our work was to design a concept and data structure for easy and semi-automated set up of Cost-Effectiveness Markovmodels for rhythmologic topics and evaluate those with a portion of available literature.
\end{abstract}

\section{Introduction}

Cost-Effectiveness Analysis becomes an important tool for Providers and Payers in establishing new therapeutic options for patients. Established tools work on a base of Markov-Chain Analysis and/or Decision Tree Models like the Software Decision Maker ${ }^{\circledR}$ or Data ${ }^{\circledR}$ [1] [2]. Recently the Models become more and more part in payor-provider negotiations or - already for a very long time-period - are relevant for internal calculations e.g. for pricing departments of pharmaceutical companies.

Normally in-house Solutions keep track of used datasets from publications in simple file-systems or more complex relational databases. This way many different resources of published data and "Clinical Evidence Data" being published on certain topics are built. This heterogeneous landscape and the fact, that Medical Databases (e.g. MedLine) do not provide the data in a fixed and searchable format lead to a huge time gap in "time to provider" and "time to payer" of this published information. At the Heart Center Leipzig the Authors build different Disease-Models with the Data ${ }^{\circledR}$ Software. Objective of this work was to design a concept and data structure for easy and semi-automated set up of CostEffectiveness Markovmodels for rhythmologic topics and evaluate those with a portion of available literature. Figure 1 shows the Vision of how the new dataformats could be shared and validated and over global medical databases.

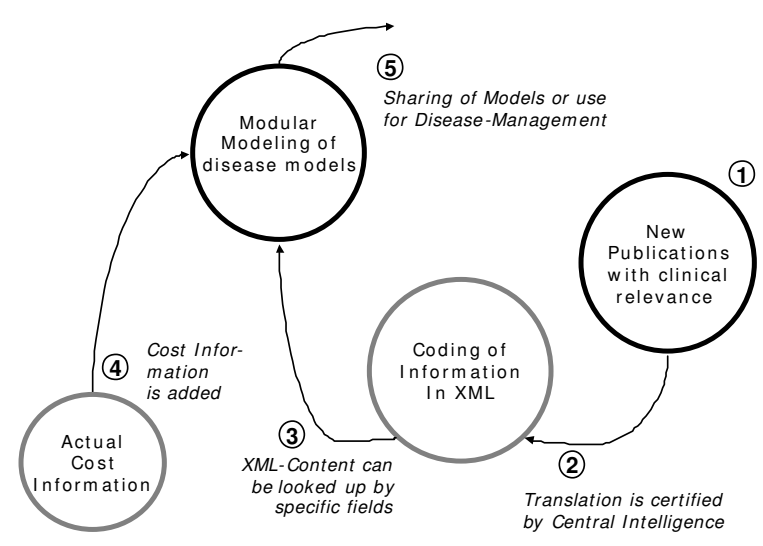

Figure 1. Vision of Sharing Clinical Evidence Data from medical Publications over XML Format

\section{Material and methods}

The Tools for Concept and Data structure were built using a XML-Designtool from Altova Inc. [3] and a Visual Basic Program. A first alpha-prototype of a markov node representation layer webfrontend with condensed reference information was built with a Tomcat / Cocoon 2.0 Framework [4]. The Framework makes it easy and possible to implement a XML-processing pipeline with each component specializing on a particular operation. This "Lego ${ }^{\circledR}$ "-like approach lets one process multiple XML-files from different resources and "glue" them together to the desired output formats like structured PDFs or visualizations of Decisionchains.

For the Evaluation of the Model we used Review / Original Literature from Carol et al. on CostEffectiveness of Ablation for Supraventricular Tachykardia and Literature from Eckman et al. and Weerasooriya et al. on Cost-Effectiveness of PAF Treatments [5] [6].

Dataentry was done over the Tool from Altova and the Prototype. The Installation could be established on a normal laptop Pentium II machine with a Windows 2000 Operating System. In a first version the Cocoon Framework could render a text-oriented view of the nodes of the built models and search and extract certain datafields / evidence from XML-files in a local directory. 


\section{First results}

As a result of our Work we developed two XMLSchemes, one to represent EBM / Cost-Effectiveness relevant information (Demographic, Clinical, Costs, Utilities and Disutilities) from Publications ( = MedEcXML-REF) and a second to map and weight this information to defined markov nodes (MedEc-XMLMAP). With the System 60 References could be mapped into the new Data structure.

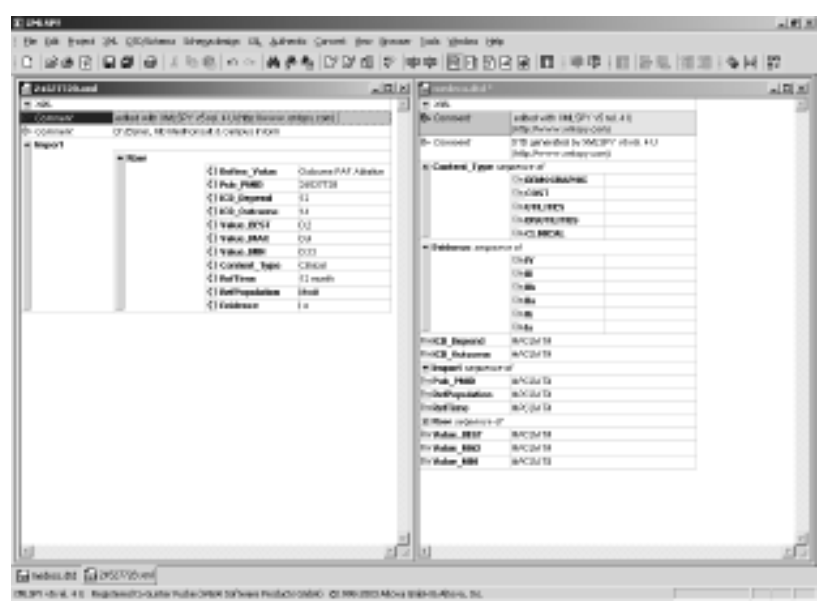

Figure 2. Altova Software XML-Spy for visualization of XML-structure

With the prototype in Tomcat / Cocoon 2.0 we could rebuild the models from the literature graphically. The System could then give a web-enabled fast overview of the underlying referenced information and Clinical Evidence of the Data per node (for the Evidence Rating we used a modified model from U.S. Preventive Services Task Force).

$<$ Define_Value $>$ Amiodarone Therapy $</$ Define_Value $>$ $<$ Pub_PMID $>24251671</$ Pub_PMID $>$

$<I C D \_$Depend $>0</ I C D \_$Depend $>$

$<$ ICD_Outcome $>0</ I C D \_O u t c o m e>$

$<$ Value BEST $>1000<$ Nalue BEST>

$<$ Value_MAX $>1200</$ Value_MAX>

$<$ Value_MIN $>900</$ Value_MIN $>$

$<$ Content_Type $>$ Costs $</$ Content_Type $>$

$<$ RefTime $>12$ month $</$ RefTime $>$

$<$ RefPopulation $>$ Post Infarction $</$ RefPopulation $>$

$<$ Evidence $>\mathrm{I} \mathrm{b}</$ Evidence $>$

Figure 3. Excerpt from a Med-Ec-XML-REF encoded reference from the Prototype
At least new data could be added fast through the integration of XML-structured data from a number of (fictive) new publications for evaluation purposes and validation of the system.

\section{Discussion}

The developed data scheme seems to be feasible to extract relevant information from the publications. Mapping the Information from the references still needs sort of interpretation which needs to be commented in both the schemes.

A further granulation of the XML schemes may therefore be necessary. The process of mapping reference information to the markov nodes is highly enhanced due to semi-automation by the use of common classification codes for procedures and outcomes.

The principle of the graphical representation may be useful for consecutive literature search / investigations being performed for further evidence of CostEffectiveness at certain points.

\section{References}

[1] See URL http://www.treeage.com

[2] See URL www.tufts-nemc.org/medicine/cdm/dmaker1.htm for Pratt Medical Software Decision Maker

[3] See URL http://www.altova.com

[4] See URL http://www.apache.org

[5] Eckman MH, Falk RH, Pauker SG. Cost-effectiveness of therapies for patients with nonvalvular atrial fibrillation. In: Arch Intern Med (United States), Aug 10-24 1998, 158(15) p1669-77

[6] Weerasooriya R, Jais P, Le Heuzey JY, et al. Cost analysis of catheter ablation for paroxysmal atrial fibrillation. In: Pacing Clin Electrophysiol (United States), Jan 2003, 26(1 Pt 2) p292-4

[7] Harris RP, Helfand M, Woolf $\mathrm{SH}$, et al. Current methods of the US Preventive Services Task Force: a review of the process. In: Am J Prev Med (Netherlands), Apr 2001, 20(3 Suppl) p21-35

Address for correspondence.

Christian Elsner

Thomasiusstr. 9

04109 Leipzig

Germany

Ch.Elsner@gmx.de 